\title{
Perspective Piece Global Health Education during the COVID-19 Pandemic: Challenges, Adaptations, and Lessons Learned
}

\author{
Kristina M. Krohn, ${ }^{1,2 *}$ Michael A. Sundberg, ${ }^{1,2}$ Nasreen S. Quadri, ${ }^{1,3}$ William M. Stauffer, ${ }^{1,2,4}$ Adriana Dhawan, ${ }^{1,2}$ \\ Hope Pogemiller, ${ }^{1,2}$ Viviane Tchonang Leuche, ${ }^{2}$ Sarah Kesler, ${ }^{1}$ Tsige H. Gebreslasse, ${ }^{1,5}$ Megan K. Shaughnessy, ${ }^{4}$ Bobbi Pritt, ${ }^{6}$ \\ Alma Habib, ${ }^{1}$ Beth Scudder, ${ }^{1}$ Sarah Sponsler, ${ }^{1}$ Stephen Dunlop, ${ }^{7}$ and Brett Hendel-Paterson ${ }^{1,5}$ \\ ${ }^{1}$ Global Medicine, Department of Medicine, University of Minnesota Medical School, Minneapolis, Minnesota; ${ }^{2}$ Department of Pediatrics, \\ University of Minnesota Medical School, Minneapolis, Minnesota; ${ }^{3}$ Internal Medicine, Allina Health, Minneapolis, Minnesota; ${ }^{4}$ Internal Medicine, \\ Hennepin Healthcare, Minneapolis, Minnesota; ${ }^{5}$ Internal Medicine HealthPartners, St. Paul, Minnesota; ${ }^{6}$ Department of Laboratory Medicine and \\ Pathology, Mayo Clinic, Rochester, Minnesota; ${ }^{7}$ Emergency Medicine, Hennepin Healthcare, Minneapolis, Minnesota
}

\begin{abstract}
Global health education programs should strive continually to improve the quality of education, increase access, create communities that foster excellence in global health practices, and ensure sustainability. The COVID-19 pandemic forced the University of Minnesota's extensive global health education programs, which includes a decade of hybrid online and in-person programing, to move completely online. We share our experience, a working framework for evaluating global health educational programming, and lessons learned. Over the decades we have moved from a predominantly passive, lecture-based, in-person course to a hybrid online (passive) course with an intensive hands-on 2-week requirement. The pandemic forced us to explore new active online learning models. We retained our on-demand, online passive didactics, which used experts' time efficiently and was widely accessible and well received. In addition, we developed a highly effective synchronous online component that we felt replaced some of the hands-on activities effectively and led us to develop new and innovative "hands-on" experiences. This new, fully online model combining quality asynchronous and synchronous learning provided many unanticipated advantages, such as increasing access while decreasing our carbon footprint dramatically. By sharing our experience, lessons learned, and resources, we hope to inspire other programs likewise to innovate to improve quality, access, community, and sustainability in global health, especially if these innovations can help decrease negative aspects of global health education such as its environmental impact.
\end{abstract}

In 2020, global health education programs, which historically have been highly dependent on international travel, were forced to adapt to sudden restrictions as a result of COVID-19. Responses ranged from halting or limiting activities to transitioning quickly to online platforms. ${ }^{1}$ As the pandemic changes and programs move forward, it is critical to consider whether some of the adaptations hold valuable lessons for improving the future of global health education. To stimulate widespread programmatic evaluation, we describe our experience adapting the University of Minnesota's extensive global health education program, including our American Society of Tropical Medicine and Hygiene (ASTM$\mathrm{H})$-accredited Global Health Course, to accommodate COVID-19 restrictions. We describe both our challenges and successes as we seek to improve educational quality, accessibility, and foster community while simultaneously decreasing our environmental impact and ensuring better use of resources. We hope these reflections will be useful for other global health programs, and will stimulate further sharing and dialogue.

\section{PRE-PANDEMIC: COURSE BEGINNINGS AND EVOLUTION}

The University of Minnesota Global Health Course was launched in 2005, consisting of 8- to 9-hour days of mostly didactic (passive) lectures, with interspersed laboratory time

*Address correspondence to Kristina Krohn, Global Medicine, Department of Medicine, University of Minnesota, 420 Delaware St. SE, MMC 741, Minneapolis, MN 55455. E-mail: kroh00040@umn. edu over 8 weeks. Recognizing the need for increased flexibility for our diverse learners, many of whom were practicing clinicians, the course transitioned to a hybrid online/in-person format in 2010. Participants watched online asynchronous didactics in advance of the course to obtain essential knowledge for full participation in the 2-week intensive hands-on course component. Although the information in our courses is rated by learners to be excellent and contains all the necessary material to prepare individuals to sit for the ASTMH CTropMed $^{\circledR}$ examination or a diploma in tropical medicine and hygiene through the Royal College of Physicians, the format of mostly passive lectures left room for improvement. Passive learning is inferior to active methods that have emerged as best practices of medical education. ${ }^{2-5}$ Like many courses, our course directors have been limited by lack of time and funding for investigating and using new instructional technologies.

Hosting the in-person component in Minnesota remained an obstacle for international participants. We partnered with Makerere University in Kampala, Uganda, and Mahidol University in Bangkok, Thailand, to improve quality and access. Learners would take our online content and use time in Thailand or Uganda for the hands-on component. Using different locations focused content on a given locale's diseases, cultures, needs, and resources, thereby providing high-quality immersive experiences. Subsidizing local and regional students increased access for learners from low- and middleincome countries (LMICs). The courses are co-created and taught by in-country expert colleagues, so our learners benefit from their experience and expertise practicing tropical medicine and global health. This approach also decreased faculty 
travel costs and improved representation of perspectives from colleagues in the "Global South."6 Regardless of location, our intensive in-person courses create a sense of community, camaraderie, and collegiality that last long past the course itself. The online sphere was different. Although we established discussion boards and e-mail listservs, we struggled to create a sense of connection pre-pandemic.

In-person training either requires travel or excludes some individuals based on geography and is ultimately detrimental to our planet's sustainability. The cost and carbon emissions of long-distance travel for experts and course participants of international conferences is recognized increasingly, as demonstrated by analyses of the ASTMH and Radiological Society of North America annual meetings. ${ }^{7,8}$ Additionally concerning is the fact that the negative effects of climate change affect LMICs disproportionately-the very populations we hope ultimately benefit from our courses..$^{9,10}$

\section{COVID-19: A FORCED OPPORTUNITY WITH A STEEP LEARNING CURVE}

As all in-person training and travel ceased in March 2020, we questioned the immediate and long-term viability of our courses. For years pre-pandemic, we strived to improve quality, access, community, and sustainability. COVID-19 threatened to remove the improvements we had achieved and highlighted our shortcomings. Although our transition to fully online content delivery was borne out of necessity, it provided an opportunity to make large-scale improvements to our program. Here we describe how our adaptations succeeded or failed based on the values of improving quality, facilitating accessibility, fostering community, and sustainability.

Maintaining and expanding high-quality online education. The pandemic accelerated our shift to and appreciation of active online learning. Having hired an academic technologist and trained faculty in andragogy and online education theories helped us create new promising practices. Smallgroup case discussions, such as the "pre- and post-travel case" provided in Supplement 1, worked well online, especially as the pandemic progressed and participants became more familiar with engaging in online video groups. Smallgroup online tabletop exercises and virtual simulations created a sense of community among learners, and taught important skills through team decision making and problembased learning, which follows best practices of adult education. $^{3-5}$ A sample virtual simulation facilitator guide and slides are shared in Supplements 2 and 3. We conducted virtual laboratories using digital microscopy paired with an interactive question-answer format. During these live sessions, the instructor projected the microscope field onto a computer screen using a digital camera and associated software. The learners viewed the instructor's screen in real time and watched as microorganisms of interest were located and analyzed. We felt this offered an improved experience over our conventional laboratory sessions during which learners rotated among microscope sessions with preconfigured displays, because the learners could now observe the process of identifying the various microorganisms. On the other hand, the virtual experience did not allow the learners the opportunity to "drive" the microscope and attempt to find the microorganisms themselves, which is another component of our in-person hands-on laboratories.
We paired these live components with asynchronous content. Asynchronous content is similar to homework; it is learning done outside of class time with teachers. We specifically used recorded lectures, readings, and interactive modules that provide high-quality content in multimedia formats that combine shorter videos, readings, and questions. An example of an interactive asynchronous module, "Cancer in Low-Resource Settings," showcasing one of our local colleagues in Tanzania, is shared in Supplement 4. Although these activities were well received, they required significant preparation, expertise, and time to create, making them demanding for our faculty.

When comparing the quality of our synchronous online sessions with our previous in-person course, our learner evaluations remained stable. Participants are asked each day to rank the days materials as excellent, very good, good, fair, or poor. In 2018, 2019, and 2021, the mode for each day with data was very good or excellent, with 12 "excellent" and seven "very good" in 2018, 13 "excellent" and five "very good" in 2019, and 17 "excellent" and three "very good" in 2021. In 2020, 2 days received a mode evaluation of "good", one "very good", and 16 "excellent." The final exam for this portion of our course is created from a question using many of the same multiple-choice questions year to year. The average scores in 2017 and 2019 when in-person was 69\% (SD, 21; $n=139)$. Final scores on the exam from 2018 were not saved. In 2020, we did not offer a final exam. In 2021, during the completely online course, the average on the final exam was $79 \%$ (SD, $17 ; n=48$ ). As a CTropMed Diploma Course, the ultimate test will be the quality of care provided by our learners and pass rates for the CTropMed exam. At the time of this writing, the CTropMed exam has not yet occurred for the learner cohorts who took our online courses, and clear quality alternative methods for evaluating medical e-learning have yet to be determined. ${ }^{11-14}$

Expanding access. Some attempts at asynchronous learning pre-pandemic grew exponentially during the pandemic as institutions around the world sought virtual education, particularly content like ours, which focuses on better health care for underserved populations. The WHO is translating our dermatology course into French, Spanish, and Portuguese to be disseminated through OpenWHO to lowresource settings later in 2021. The University of Minnesota, like many other universities, removed all students from clinical activities early during the COVID-19 pandemic to save personal protective equipment for fully trained personnel and to protect learners. As a result, the medical school, like many medical schools around the world, rapidly sought elective virtual curricula. ${ }^{15-23}$ When the university asked for virtual electives, we as faculty in global medicine rapidly converted our online curriculum designed for continuing medical education to instruct more than 120 medical students on immigrants and refugee health, global health, and tropical medicine. In-person courses could never have been expanded in these ways. As students have returned to in-person clinical settings to complete their required clinical rotations, we have maintained some of the online elective options. Students continue to choose the virtual electives that help them learn to care better for our diverse communities, with 50 enrollments in our electives in the first half of the 2021-2022 schoolyear. 
Virtual learning spaces removed the barriers of distance and travel. Colleagues could join and provide their perspectives from across the United States, and countries including Panama, Thailand, Jordan, the United Kingdom, Australia, New Zealand, Gabon, Kenya, and Tunisia. Often, the people best suited to teach were deeply involved in clinical or public health interventions related directly to the pandemic and did not have time to travel, but could attend a video meeting. Sometimes experts involved in COVID-19 responses could not even meet during typical class times, but did have time to record a lecture or share a personal story. We scheduled 2 synchronous hours and 2 asynchronous hours per day for 4 weeks rather than our traditional 2 weeks of 8-hour days. Participants appreciated the flexibility, the shortened days, and the longer duration, as many said they would not have been able to take the time off to attend a full-day, week-long course, but could adjust clinic schedules for a few hours each day over the month. We recorded most of the online discussions and were able to select, edit, and share highyield recordings with those who could not attend synchronously. It was a good reminder that passive learning may be appropriate depending on the context and content, in particular when it increases access.

Difficulties with Internet bandwidth and time zones prevented some participants' full participation. Most sessions in 2020 and in 2021 had attendance rates of more than $80 \%$, with all students in 2021 being able to attend a minimum of 15 of the 20 synchronous days. We do not know how many people chose not to participate because of time zone constraints. Interactive video sessions required greater bandwidth and excluded some participants, although some adaptations could be made by using audio only. Other participants were limited by time zone differences. Prerecorded sessions required lower bandwidth, were more accessible, and were valued by course participants in lower resource settings who may otherwise have been excluded from participating in our course.

We noted that the online environment allowed greater geographic representation in both synchronous and asynchronous activities, leading to broader representation of colleagues from the Global South as educators. Time, travel, and financial barriers may have made their participation in a U.S.-based course more difficult. In one example, colleagues at partner sites in Laos, Tanzania, India, and the United States participated in a very well-received synchronous panel discussion. International instructors also gave lectures over Zoom. The "digital divide" remained, however, and at times we struggled to accommodate slow Internet connections. We adapted by having instructors forward slides for us to display, and by accessing the video sessions through voice-only options when needed.

One value that we have not and cannot adequately address in this article is the desire to be more equitable in our global health programs. To be more equitable, we must address the colonial history of global health and tropical medicine, and we must acknowledge the ways in which many of our current models perpetuate inequities. Evaluating educational interventions based on equity requires defining who the audience is and who has the right to determine what is considered equitable. Moving online is insufficient to make global health education equitable, and it is beyond the scope of this article to discuss true equity. Therefore, we focused on improving access. We have offered significant discounts to our courses for participants from LMICs, waived fees and supported learners from our partner sites, and sponsored travel to Minnesota for LMIC colleagues to attend our course. Moving fully online could potentially decrease costs further for participants-both the cost of the course and removing the cost of travel, which can increase access dramatically. For our usual in-person course in preCOVID years, we averaged spending around $\$ 10,000$ on travel for speakers. This does not include waiving the course fee and supporting travel and accommodations to make it possible for learners from LMICs to attend the course. Because we had invested previously in hiring an academic technologist for our courses, the University of Minnesota already had a Zoom contract, and we had previously dedicated faculty time to online education, these costs remained fixed for 2020 and 2021.

Fostering community. One of our biggest fears in moving our Global Health Course fully online was the loss of community. However, seeing our colleagues in their homes, with their families coming in and out of the screen, added a new dimension to our lives. Virtual meetings forced many of us to share our private lives with our colleagues in different, often beneficial, ways. Many of us who previously kept work and family separate now bonded over the shenanigans of children or pets vying for more attention. Leaving Zoom meetings open after class time officially ended, and having open break-out rooms that participants could use for more individual conversations, and side bar chats all helped encourage some of the side conversations that happen in person. These interactions do not replace completely hallway conversations or the discussions shared during meals; however, we were surprised by the depth of community we were able to create.

Another pleasant surprise was the ability to create some community online through small and large groups. We kept our class size to less than 50 individuals to ensure that participants could ask questions and start discussions with the experts. Participants and experts from around the world were able to share the similarities and differences in their experiences in real time, and a number of our participants bonded and planned to collaborate on future endeavors.

Sustainability and environmental responsibility. Lack of global or domestic travel to our courses led to a much lower environmental impact. For our in-person course in 2019, there were 38 traveling participants-one international speaker, 10 domestic speakers, nine international participants, and 18 domestic participants from outside of Minnesota-compared with zero traveling participants in 2020 and 2021. Being online eliminated travel and decreased time demands on experts in the United States and from around the world, which was particularly important for updates related to ongoing COVID-19 outbreaks in different locales. Our asynchronous online content is enduring material, which can be improved iteratively over time. Our synchronous "live" sessions, although more time- and faculty-intensive to create, will serve as a base for leading similar sessions in the future.

\section{MOVING INTO THE FUTURE}

We acknowledge that online education is different from in-person learning, and there is still great disparity in online 
TABLE 1

Benefits and drawbacks of online education in global health as identified through discussions and experience of University of Minnesota Global Health and Tropical Medicine Curriculum faculty, 2020 through 2021

\begin{tabular}{ll}
\hline \multicolumn{1}{c}{ Benefits of online education } & Drawbacks of online education \\
\hline $\begin{array}{l}\text { Technology investments result in novel teaching methods. } \\
\text { Allows learners to select education courses from around the world, } \\
\text { not just in their physical location. }\end{array}$ & $\begin{array}{c}\text { There is a lack of an in-person community. } \\
\text { It is exclusionary based on Internet bandwidth. }\end{array}$ \\
$\begin{array}{l}\text { Learners can customize their education. } \\
\text { Learning can be self-paced for individual learning styles and can } \\
\text { accommodate those with busy schedules. }\end{array}$ & $\begin{array}{c}\text { It is exclusionary based on time zones. } \\
\text { Some benefits are lost from in-person, hands-on mentored } \\
\text { experiences (physical skills in the laboratory and procedures). }\end{array}$ \\
$\begin{array}{l}\text { Flexible asynchronous activities can increase access for diverse } \\
\text { participants significantly. }\end{array}$ & $\begin{array}{c}\text { The creation of interactive materials is intensive in terms of time } \\
\text { and technology. }\end{array}$ \\
$\begin{array}{l}\text { One is able to increase involvement from international/remote } \\
\text { faculty in live virtual sessions. }\end{array}$ & $\begin{array}{c}\text { Assessing the quality of education is difficult until after significant } \\
\text { amount of time and investment. }\end{array}$ \\
$\begin{array}{l}\text { Material may be reused and shared easily and broadly when } \\
\text { appropriate. }\end{array}$ & $\begin{array}{c}\text { Up-front investment and different skill sets are required to create } \\
\text { quality online learning. }\end{array}$ \\
$\begin{array}{l}\text { There is the potential to decrease costs for participants. } \\
\text { There is decreased environmental impact. }\end{array}$ & $\begin{array}{c} \\
\text { Assessing competence requires different techniques. }\end{array}$ \\
\hline
\end{tabular}

education as a result of the digital divide, particularly for learners without high-speed Internet access. We found new challenges were introduced by accounting for learners in multiple time zones when planning live virtual activities. We are now critically analyzing the benefits, drawbacks, challenges, and solutions for improving online global health education. We summarize these in Tables 1 and 2 in the hopes others can learn from our experience.

Although the pandemic has taken, and continues to take, a terrible toll, its disruption has forced innovation in global health education and galvanized investment in online education methodologies. We have learned that investing time, energy, and people has the potential to increase flexible access dramatically to quality online education and can better meet best practices for adult education while decreasing our carbon footprint. At the University of Minnesota, we are exploring new hybrid and fully virtual course models. We are seeking regional collaborations for hands-on courses that build local capacity by highlighting local faculty, that improve access through local scholarships, and that increase community and mutual benefit through matching students from high-income countries with students from LMICs. In addition, we are investing time and energy into both innovative active online learning and lower bandwidth online options to improve quality and access further for all our colleagues. We hope to collaborate with ASTMH-accredited courses and others to create, share, and disseminate quality teaching materials as the online materials are easier to share among institutions. We hope our experience is helpful to all global health educators who are critically evaluating their own programs with the intent of maximizing quality and access for all, thereby supporting those who seek to learn, teach, and practice global medicine in ways that promote sustainable global community.

Received July 10, 2021. Accepted for publication September 15, 2021.

Published online October 11, 2021.

Note: Supplemental materials appear at www.ajtmh.org.

Acknowledgments: Thank you to all the faculty and participants who make these courses possible. Well over 150 faculty members have shared their expertise through our courses. Special thanks to Drs. Randy Hurley at HealthPartners in Minnesota and Furaha Serventi at Kilimanjaro Christian Medical Centre in Tanzania for their assistance with the interactive oncology module shared as a supplement. The American Society of Tropical Medicine and Hygiene has waived the Open Access fee for this article due to the ongoing COVID-19 pandemic.

Financial support: This work was supported by the University of Minnesota COVID-19 Educational Innovation Grant.

Disclosure: The University of Minnesota Global Medicine program is funded through registration fees from course participants, institutional

TABLE 2

Challenges for the future of online global health education, with potential solutions as identified through discussions and experience of the University of Minnesota Global Health and Tropical Medicine Curriculum faculty, 2020 through 2021

\begin{tabular}{|c|c|}
\hline Challenges for the future & Proposed solutions \\
\hline $\begin{array}{l}\text { Faculty/participants may be inexperienced with technologies or } \\
\text { learning modalities. }\end{array}$ & $\begin{array}{l}\text { Have dedicated staff or faculty prep experts for different } \\
\text { technologies used. } \\
\text { Conduct practice run-throughs before live sessions, with an eye } \\
\text { on video and audio quality. }\end{array}$ \\
\hline $\begin{array}{l}\text { Asynchronous interactive modules take time and investment to } \\
\text { create. }\end{array}$ & $\begin{array}{l}\text { Invest in training staff and faculty in creating quality interactive } \\
\text { online content. }\end{array}$ \\
\hline Need variety to prevent "Zoom fatigue." & $\begin{array}{l}\text { Invest in instructional design to improve usability and variety for } \\
\text { participants. }\end{array}$ \\
\hline Manage multiple time zones. & Select timing carefully to ensure synchronous content. \\
\hline $\begin{array}{l}\text { A "digital divide" limits access for participants/partner faculty with } \\
\text { low bandwidth. }\end{array}$ & $\begin{array}{l}\text { Invest in digital infrastructure for partners. } \\
\text { Support worldwide Internet access. } \\
\text { Share slides before sessions; include audio-only access. }\end{array}$ \\
\hline Improve worldwide representation of experts. & $\begin{array}{l}\text { Invest and train staff and faculty at partner sites in creating quality } \\
\text { online education. } \\
\text { Create templates and clear instructions for experts, highlighted } \\
\text { in online materials, to make participation as easy as possible. }\end{array}$ \\
\hline
\end{tabular}


agreements, and support from the University of Minnesota. W. M. S. has royalties for an UpToDate chapter and a one-time honorarium for educational consultation to Fishawack/BioEmergent Technologies.

Authors' addresses: Kristina Krohn, Michael A. Sundberg, Adriana Dhawan, and Hope Pogemiller, Global Medicine, Department of Medicine, University of Minnesota Medical School, Minneapolis, MN, and Department of Pediatrics, University of Minnesota Medical School, Minneapolis, MN, E-mails: kroh00040@umn.edu, msundberg@umn.edu, dhawa027@umn.edu, and poge0008@umn. edu. Nasreen S. Quadri, Global Medicine, Department of Medicine, University of Minnesota Medical School, Minneapolis, MN, and Internal Medicine, Allina Health, Minneapolis, MN, E-mail: quadr015@umn.edu. William M. Stauffer, Global Medicine, Department of Medicine, University of Minnesota Medical School, Minneapolis, MN, Department of Pediatrics, University of Minnesota Medical School, Minneapolis, MN, and Internal Medicine, Hennepin Healthcare, Minneapolis, MN, E-mail: stauf005@umn.edu. Viviane Tchonang Leuche, Department of Pediatrics, University of Minnesota Medical School, Minneapolis, MN, E-mail: tchon002@umn.edu. Sarah Kesler, Alma Habib, Beth Scudder, and Sarah Sponsler, Global Medicine, Department of Medicine, University of Minnesota Medical School, Minneapolis, MN, E-mails: kesle002@umn.edu, habib043@umn.edu, bscudder@umn.edu, and radic011@umn.edu. Tsige H. Gebreslasse and Brett Hendel-Paterson, Global Medicine, Department of Medicine, University of Minnesota Medical School, Minneapolis, MN, and Internal Medicine HealthPartners, St. Paul, MN, E-mails: tgebrela@umn.edu and bhendel@umn.edu. Megan K. Shaughnessy, Internal Medicine, Hennepin Healthcare, Minneapolis, MN, E-mail: megan.shaughnessy@hcmed.org. Bobbi Pritt, Department of Laboratory Medicine and Pathology, Mayo Clinic, Rochester, MN, E-mail: pritt.bobbi@mayo.edu. Stephen Dunlop, Emergency Medicine, Hennepin Healthcare, Minneapolis, MN, E-mail: stephen.dunlop@hcmed.org.

This is an open-access article distributed under the terms of the Creative Commons Attribution (CC-BY) License, which permits unrestricted use, distribution, and reproduction in any medium, provided the original author and source are credited.

\section{REFERENCES}

1. Weine $S$ et al., 2021. Global health education amidst COVID-19: disruptions and opportunities. Ann Glob Health 87: 1-6.

2. Reed $S$ et al., 2014. Applying adult learning practices in medical education. Curr Probl Pediatr Adolesc Health Care 44: 170-181.

3. Roddy C, Amiet DL, Chung J, Holt C, Shaw L, McKenzie S, Garivaldis F, Lodge JM, Mundy ME, 2017. Applying best practice online learning, teaching, and support to intensive online environments: an integrative review. Front Educ 2: 59.

4. Means B, Toyama Y, Murphy R, Bakia M, Jones K, 2010. Evaluation of Evidence-Based Practices in Online Learning: A Metaanalysis and Review of Online Learning Studies Center for Technology in Learning. Available at: www.ed.gov/about/ offices/list/opepd/ppss/reports.html. Accessed June 11, 2021.

5. Hanover Research Council, 2009. Best Practices in Online Teaching Strategies. Available at: http://www.ihep.org/. Accessed June 11, 2021.

6. Velin L, Lartigue J-W, Johnson SS, Zorigtbaater A, Kanmounye US, Truche P, Joseph MN, 2021. Conference equity in global health: a systematic review of factors impacting LMIC representation at global health conferences. BMJ Glob Health 6: e003455

7. Bousema T, Selvaraj P, Djimde AA, Yakar D, Hagedorn B, Pratt A, Barret D, Whitfield K, Cohen JM, 2020. Perspective piece reducing the carbon footprint of academic conferences: the example of the American Society of Tropical Medicine and Hygiene. Am J Trop Med Hyg 103: 1758-1761.

8. Yakar D, Kwee TC, 2020. Carbon footprint of the RSNA annual meeting. Eur J Radiol 125: 108869.

9. Watts $\mathrm{N}$ et al., 2019. The 2019 report of The Lancet Countdown on health and climate change: ensuring that the health of a child born today is not defined by a changing climate. Lancet 394: 1836-1878.

10. Tang L, li R, Tokimatsu K, Itsubo N, 2018. Development of human health damage factors related to $\mathrm{CO} 2$ emissions by considering future socioeconomic scenarios. Int $J$ Life Cycle Assess 23: 2288-2299.

11. de Leeuw R, de Soet A, van der Horst S, Walsh K, Westerman M, Scheele F, 2019. How we evaluate postgraduate medical e-learning: systematic review. JMIR Med Educ 5: e13128.

12. de Leeuw R, Westerman M, Walsh K, Scheele F, 2019. Development of an instructional design evaluation survey for postgraduate medical e-learning: content validation study. $J$ Med Internet Res 21: e13921.

13. Sinclair P, Kable A, Levett-Jones T, 2015. The effectiveness of Internet-based e-learning on clinician behavior and patient outcomes: a systematic review protocol. JBI Database Syst Rev Implement Reports 13: 52-64.

14. Taveira-Gomes T, Ferreira P, Taveira-Gomes I, Severo M, Ferreira MA, 2016. What are we looking for in computer-based learning interventions in medical education? A systematic review. J Med Internet Res 18: e204.

15. Roskvist R, Eggleton K, Goodyear-Smith F, 2020. Provision of e-learning programmes to replace undergraduate medical students' clinical general practice attachments during COVID-19 stand-down. Educ Prim Care 31: 247-254.

16. Lazzereschi L, Kirtley D, 2021. Provision of e-learning programmes to replace undergraduate medical students' clinical general practice attachments during COVID-19 stand-down: a student perspective. Educ Prim Care 32: 63.

17. Alsoufi $A$ et al., 2020. Impact of the COVID-19 pandemic on medical education: medical students' knowledge, attitudes, and practices regarding electronic learning. PLoS One 15: e0242905.

18. Sandhu P, de Wolf $M, 2020$. The impact of COVID-19 on the undergraduate medical curriculum. Med Educ Online 25: 1764740.

19. Ahmed SA, Hegazy NN, Abdel Malak HW, Kayser WC III, Elrafie NM, Hassanien M, Al-Hayani AA, El Saadany SA, Ai-Youbi $A O$, Shehata $M H, 2020$. Model for utilizing distance learning post COVID-19 using (PACT) ${ }^{\mathrm{TM}}$ a cross sectional qualitative study. BMC Med Educ 20: 400.

20. Coe TM, McBroom TJ, Brownlee SA, Regan K, Bartels S, Saillant N, Yeh H, Petrusa E, Dageforde LA, 2021. Medical students and patients benefit from virtual non-medical interactions due to COVID-19. J Med Educ Curric Dev 8: 23821205211028343

21. Nowell L, Lorenzetti D, Jacobsen M, Lorenzetti L, Paolucci EO, 2021. Translating caring competencies to remote working environments: a systematic review protocol. BMJ Open 11: e048459.

22. Chasset F, Barral M, Steichen O, Legrand A, 2021. Immediate consequences and solutions used to maintain medical education during the COVID-19 pandemic for residents and medical students: a restricted review. Postgrad Med J.

23. Stout RC, Roberts S, Maxwell-Scott H, Gothard P, 2021. Necessity is the mother of invention: how the COVID-19 pandemic could change medical student placements for the better. Postgrad Med J 97: 417-422. 